\title{
Energetics and Mechanism of Decomposition of Trifluoromethanol
}

\author{
Minh Tho Nguyen, ${ }^{\mathrm{a}, \mathrm{b}}$ Myrna H. Matus, ${ }^{\mathrm{a}}$ Vu Thi Ngan, ${ }^{\mathrm{b}}$ Ralf Haiges, ${ }^{\mathrm{c}}$ Karl O. Christe, ${ }^{\mathrm{c}}$ \\ and David A. Dixon $\left.{ }^{\mathrm{a},},\right\rangle^{\dagger}$ \\ a Department of Chemistry, The University of Alabama, Shelby Hall, Tuscaloosa, Alabama \\ $35487-0336$ \\ ${ }^{\mathrm{b}}$ Department of Chemistry, University of Leuven, B-3001 Leuven, Belgium \\ ${ }^{\mathrm{c}}$ Loker Research Institute and Department of Chemistry, University of Southern \\ California, Los Angeles, CA 90089-1661
}

Supporting Information Available: Total MP2 and $\operatorname{CCSD}(\mathrm{T})$ energies $\left(E_{\mathrm{h}}\right)$ as a function of basis set extrapolated to the complete basis set limit. MP2 vibrational modes $\left(\mathrm{cm}^{-1}\right)$. Skodje \& Truhlar tunneling corrections $\left(Q_{\mathrm{ST}}\right)$. Rate constants with and without $Q_{\text {sT. }}$ Symmetry and Cartessian coordinates of MP2/aVTZ optimized geometries.

\footnotetext{
${ }^{\dagger}$ Email: dadixon@bama.ua.edu
} 
Table S1. Total MP2 and CCSD(T) energies $\left(E_{\mathrm{h}}\right)$ as a function of basis set extrapolated to the complete basis set limit. ${ }^{\mathrm{a}}$

\begin{tabular}{|c|c|c|c|c|c|c|c|c|}
\hline \multirow{2}{*}{ Molecule } & \multicolumn{4}{|c|}{$\mathrm{MP} 2 / \mathrm{aVnZ}$} & \multicolumn{4}{|c|}{$\mathrm{CCSD}(\mathrm{T}) / \mathrm{aV} n \mathrm{Z}$} \\
\hline & $n=\mathrm{D}$ & $n=\mathrm{T}$ & $n=\mathrm{Q}$ & CBS(DTQ) & $n=\mathrm{D}$ & $n=\mathrm{T}$ & $n=\mathrm{Q}$ & CBS(DTQ) \\
\hline $\operatorname{HF}\left({ }^{1} \Sigma_{\mathrm{g}}\right)$ & -100.255804 & -100.340891 & -100.369761 & -100.386332 & -100.263641 & -100.349577 & -100.377384 & -100.393207 \\
\hline $\mathrm{HF}$ dimer $\left({ }^{1} \mathrm{~A}^{\prime}\right)$ & -200.519075 & -200.689285 & -200.746885 & -200.779931 & -200.534943 & -200.706845 & -200.762253 & -200.793756 \\
\hline $\mathrm{HF}$ trimer $\left({ }^{1} \mathrm{~A}^{\prime}\right)^{\mathrm{b}}$ & -300.792046 & -301.047316 & -301.133673 & -301.183214 & -300.816136 & -301.073938 & -301.160294 & -301.209836 \\
\hline $\mathrm{CF}_{3} \mathrm{OH}\left({ }^{1} \mathrm{~A}^{\prime}\right)$ & -412.643850 & -412.991332 & -413.106565 & -413.172434 & -412.680972 & -413.032777 & -413.143389 & -413.205980 \\
\hline $\mathrm{CF}_{3} \mathrm{O}^{\bullet}\left({ }^{2} \mathrm{~A}^{\prime}\right)$ & & & & & -411.989007 & -412.334257 & -412.442747 & -412.504129 \\
\hline $\mathrm{CF}_{3} \mathrm{O}^{+}\left({ }^{3} \mathrm{~A}_{1}\right)$ & & & & & -411.502995 & -411.843796 & -411.948974 & -412.008271 \\
\hline $\mathrm{CF}_{3} \mathrm{O}^{-}\left({ }^{1} \mathrm{~A}_{1}\right)$ & & & & & -412.151802 & -412.497144 & -412.607633 & -412.670367 \\
\hline $\mathrm{CF}_{3} \mathrm{OH}^{\circ+}\left({ }^{2} \mathrm{~A}^{\prime \prime}\right)$ & & & & & -412.207226 & -412.554972 & -412.662643 & -412.723385 \\
\hline $\mathrm{CF}_{3} \mathrm{OH}_{2}{ }^{+}\left({ }^{1} \mathrm{~A}^{\prime}\right)$ & & & & & -412.923662 & -413.278920 & -413.389022 & -413.451147 \\
\hline $\mathrm{HF}-\mathrm{CF}_{2} \mathrm{OH}^{+}\left({ }^{1} \mathrm{~A}\right)$ & & & & & -412.934019 & -413.286246 & -413.396736 & -413.459230 \\
\hline $\mathrm{CF}_{2} \mathrm{OH}^{+}-\mathrm{FH}\left({ }^{1} \mathrm{~A}^{\prime}\right)$ & & & & & -412.941989 & -413.295118 & -413.405549 & -413.467970 \\
\hline $\mathrm{CF}_{3}\left({ }^{2} \mathrm{~A}_{1}\right)$ & & & & & -336.916683 & -337.201224 & -337.290789 & -337.341482 \\
\hline $\mathrm{CF}_{2} \mathrm{O}\left({ }^{1 \mathrm{a}}{ }_{1}\right)$ & -312.378438 & -312.637285 & -312.723983 & -312.773630 & -312.403493 & -312.666657 & -312.749712 & -312.796745 \\
\hline $\mathrm{CF}_{2} \mathrm{O}^{\cdot+}\left({ }^{2} \mathrm{~B}_{2}\right)$ & & & & & -311.932486 & -312.192292 & -312.272748 & -312.318138 \\
\hline
\end{tabular}




\begin{tabular}{|c|c|c|c|c|c|c|c|c|}
\hline $\mathrm{CF}_{3} \mathrm{OH}$-ts $\left({ }^{1} \mathrm{~A}^{\prime}\right)$ & -412.572346 & -412.918525 & -413.033401 & -413.099074 & -412.605960 & -412.956453 & -413.066552 & -413.128841 \\
\hline $\mathrm{CHF}_{2} \mathrm{OH}\left({ }^{1} \mathrm{~A}^{\prime}\right)$ & -313.572109 & -313.838346 & -313.926413 & -313.976730 & -313.608072 & -313.877007 & -313.960860 & -314.008231 \\
\hline $\mathrm{CHF}_{2} \mathrm{OH}-\mathrm{ts}\left({ }^{1} \mathrm{~A}\right)$ & -313.502330 & -313.767129 & -313.854909 & -313.905082 & -313.535649 & -313.803123 & -313.886529 & -313.933647 \\
\hline $\mathrm{CH}_{2} \mathrm{FOH}\left({ }^{1} \mathrm{~A}\right)$ & -214.493356 & -214.679474 & -214.740498 & -214.775307 & -214.528310 & -214.715556 & -214.772805 & -214.805020 \\
\hline $\mathrm{CH}_{2} \mathrm{FOH}$-ts $\left({ }^{1} \mathrm{~A}\right)$ & -214.418814 & -214.603200 & -214.663861 & -214.698485 & -214.452285 & -214.637737 & -214.694449 & -214.726364 \\
\hline $\operatorname{dim}-6^{b}$ & -825.299452 & -825.992126 & -826.222027 & -826.353462 & -825.372420 & -826.075304 & -826.305205 & -826.436640 \\
\hline $\operatorname{dim}-6 t s^{b}$ & -825.254280 & -825.946179 & -826.175010 & -826.305749 & -825.323008 & -826.025017 & -826.253849 & -826.384587 \\
\hline $\operatorname{dim}-8^{b}$ & -825.300197 & -825.992266 & -826.221946 & -826.353252 & -825.373348 & -826.075590 & -826.305270 & -826.436577 \\
\hline $\operatorname{dim}-8 t s^{b}$ & -825.249721 & -825.939200 & -826.167847 & -826.298544 & -825.317244 & -826.016784 & -826.245431 & -826.376128 \\
\hline $\mathrm{CF}_{3} \mathrm{OH}-\mathrm{HF}^{\mathrm{b}}$ & -512.910157 & -513.341429 & -513.485195 & -513.567452 & -512.954662 & -513.391907 & -513.535673 & -513.617931 \\
\hline $\mathrm{CF}_{3} \mathrm{OH}-\mathrm{HFts}{ }^{\mathrm{b}}$ & -512.868405 & -513.300007 & -513.442976 & -513.524681 & -512.908921 & -513.346731 & -513.489699 & -513.571405 \\
\hline $\mathrm{CF}_{3} \mathrm{OH}-2 \mathrm{HF}^{\mathrm{b}}$ & -613.181796 & -613.697936 & -613.870236 & -613.968845 & -613.234323 & -613.757245 & -613.929545 & -614.028153 \\
\hline $\mathrm{CF}_{3} \mathrm{OH}-2 \mathrm{HFts}^{\mathrm{b}}$ & -613.147540 & -613.665187 & -613.836115 & -613.933743 & -613.195664 & -613.720373 & -613.891302 & -613.988929 \\
\hline $\operatorname{dim}-\mathbf{H} F^{b}$ & -925.570478 & -926.347352 & -926.605654 & -926.753374 & -925.650744 & -926.439364 & -926.697666 & -926.845386 \\
\hline $\operatorname{dim}-H F t^{b}$ & -925.532387 & -926.310087 & -926.566922 & -926.713620 & -925.608619 & -926.397357 & -926.654192 & -926.800891 \\
\hline $\operatorname{dim}-2 \mathbf{H} \mathbf{F}^{b}$ & -1025.836939 & -1026.698281 & -1026.985080 & -1027.149141 & -1025.926168 & -1026.799269 & -1027.086068 & -1027.250129 \\
\hline $\operatorname{dim}-\mathrm{HFts}^{\mathrm{b}}$ & -1025.782740 & -1026.644567 & -1026.929575 & -1027.092406 & -1025.864997 & -1026.738930 & -1027.023938 & -1027.186769 \\
\hline
\end{tabular}


${ }^{a}$ All energies, given in hartree, were based on MP2/aug-cc-pVTZ optimized geometries. The CCSD(T)/CBS(DTQ) energy was extrapolated by using Equation (1) with the aug-cc-pVnZ basis sets, where $n=\mathrm{D}, \mathrm{T}$ and $\mathrm{Q}^{\mathrm{b}}{ }^{\mathrm{b}} \mathrm{CCSD}(\mathrm{T}) /$ aug-cc-pVQZ and CBS energies obtained with: $\Delta \mathrm{E}[\mathrm{CCSD}(\mathrm{T}) / \mathrm{CBS}]=\Delta \mathrm{E}[\mathrm{CCSD}(\mathrm{T}) / \mathrm{aVTZ}]+(\Delta \mathrm{E}[\mathrm{MP} 2 / \mathrm{aVQZ}]-\Delta \mathrm{E}[\mathrm{MP} 2 / \mathrm{aVTZ}])$ and $\Delta \mathrm{E}[\mathrm{CCSD}(\mathrm{T}) / \mathrm{CBS}]=$ $\Delta \mathrm{E}[\mathrm{CCSD}(\mathrm{T}) / \mathrm{aVTZ}]+(\Delta \mathrm{E}[\mathrm{MP} 2 / \mathrm{CBS}]-\Delta \mathrm{E}[\mathrm{MP} 2 / \mathrm{aVTZ}])$. 
Table S2. MP2/aug-cc-pVTZ harmonic vibrational frequencies $\left(\mathrm{cm}^{-1}\right){ }^{a}$

\begin{tabular}{|c|c|}
\hline Molecule & Vibrational Modes \\
\hline $\mathrm{HF}\left({ }^{1} \Sigma_{\mathrm{g}}\right)$ & $\sigma_{\mathrm{g}}: 4122.9$ \\
\hline $\mathrm{HF}$ dimer $\left({ }^{1} \mathrm{~A}{ }^{\prime}\right)$ & a': 159.1, 217.5, 577.0, 3989.4, 4082.0; a”: 472.9 \\
\hline HF trimer $\left({ }^{1} A^{\prime}\right)$ & a’: 213.0, 989.6, 3712.1; a": 702.3; e’: 193.2, 609.8, 3832.0; e”: 507.2 \\
\hline $\mathrm{CF}_{3} \mathrm{OH}\left({ }^{1} \mathrm{~A}^{\prime}\right)$ & a': 441.9, 603.3, 636.8, 906.5, 1123.2, 1302.0, 1421.4, 3829.8; a”: 234.8, 450.3, 622.9, 1190.8 \\
\hline $\mathrm{CF}_{3} \mathrm{O}^{\bullet}\left({ }^{2} \mathrm{~A}^{\prime}\right)$ & a': 416.2, 586.2, 624.7, 904.2, 1233.9, 1298.3; a": 244.2, 602.8, 1241.1 \\
\hline $\mathrm{CF}_{3} \mathrm{O}^{+}\left({ }^{3} \mathrm{~A}_{1}\right)$ & $a_{1}: 124.8,697.2,1037.5 ;$ e: $188.0,587.5,1658.8$ \\
\hline $\mathrm{CF}_{3} \mathrm{O}^{-}\left({ }^{1} \mathrm{~A}_{1}\right)$ & $\mathrm{a}_{1}: 586.3,793.4,1641.6 ; \mathrm{e:} 410.0,562.0,900.2$ \\
\hline $\mathrm{CF}_{3} \mathrm{OH}^{\cdot+}\left({ }^{2} \mathrm{~A}^{\prime \prime}\right)$ & a': 331.0, 401.7, 571.6, 750.5, 941.1, 1016.1, 1511.0, 3486.7; a”: 106.8, 328.0, 573.7, 1447.9 \\
\hline $\mathrm{CF}_{3} \mathrm{OH}_{2}^{+}\left({ }^{1} \mathrm{~A}^{\prime}\right)$ & a': $378.6,525.4,580.6,731.2,796.9,1031.1,1415.0,1647.4,3573.4 ;$ a”: 137.3, 396.8, 571.5, 1018.6, $1478.6,3667.6$ \\
\hline $\mathrm{HF}-\mathrm{CF}_{2} \mathrm{OH}^{+}\left({ }^{1} \mathrm{~A}\right)$ & a: $95.8,111.7,164.0,176.7,263.8,565.2,597.4,649.9,776.7,1059.8,1165.3,1637.5,1762.2,3620.7,4010.7$ \\
\hline $\mathrm{CF}_{2} \mathrm{OH}^{+} \mathrm{FH}\left({ }^{1} \mathrm{~A}^{\prime}\right)$ & a': $84.6,280.1,452.0,595.2,639.5,1054.7,1324.9,1605.3,1786.2 ; 2800,4,3934.1 ;$ a'’ $94.3,131.4,803.6,987.3$ \\
\hline $\mathrm{CF}_{3}\left({ }^{2} \mathrm{~A}_{1}\right)$ & $\mathrm{a}_{1}: 710.1,1108.5 ; \mathrm{e}: 511.8,1274.7$ \\
\hline $\mathrm{CF}_{2} \mathrm{O}\left({ }^{1} \mathrm{~A}_{1}\right)$ & $a_{1}: 583.3,969.4,1951.4 ; b_{1}: 781.7 ; b_{2}: 617.6,1248.2$ \\
\hline $\mathrm{CF}_{2} \mathrm{O}^{\cdot+}\left({ }^{2} \mathrm{~B}_{2}\right)$ & $a_{1}: 569.7,1050.8,1675.2 ; b_{1}: 779.6 ; b_{2}: 491.5,1639.4$ \\
\hline $\mathrm{CF}_{3} \mathrm{OH}$-ts $\left({ }^{1} \mathrm{~A}^{\prime}\right)$ & a': $1740.7 i, 367.7,563.0,711.6,883.1,993.6,1651.1,2074.7 ;$ a”: 255.9, 570.9, 892.6, 1342.0 \\
\hline $\mathrm{CHF}_{2} \mathrm{OH}\left({ }^{1} \mathrm{~A}^{\prime}\right)$ & a': $506.1,651.1,997.8,1163.4,1347.8,1454.4,3119.4,3744.2 ;$ a": $376.1,547.2,1015.5,1384.0$ \\
\hline
\end{tabular}


$\mathrm{CHF}_{2} \mathrm{OH}$-ts $\left({ }^{1} \mathrm{~A}\right) \quad$ a: $1748.3 i, 249.3,609.1,669.8,700.6,877.0,1155.2,1199.5,1373.0,1575.3,2035.7,3205.9$

$\mathrm{CH}_{2} \mathrm{FOH}\left({ }^{1} \mathrm{~A}\right) \quad$ a: $381.4,540.9,1022.5,1077.9,1150.5,1279.6,1403.1,1458.3,1558.3,3100.5,3195.6,3838.6$

$\mathrm{CH}_{2} \mathrm{FOH}$-ts $\left({ }^{1} \mathrm{~A}\right) \quad$ a: $1771.0 i, 566.4,679.0,696.8,975.3,1253.9,1341.9,1417.8,1524.5,1668.3,2003.3,3171.6$

dim-6 a: $15.6,20.1,31.1,52.2,95.6,124.7,345.4,391.4,428.7,436.0,468.3,532.4,588.0,595.9,603.9,611.7,615.3,626.0$, $869.8,881.5,1088.7,1118.3,1140.5,1183.2,1274.8,1320.2,1402.8,1446.0,3695.9,3765.0$

dim-6ts $\quad$ a: $1413.5 i, 23.6,41.6,85.5,94.7,265.0,302.2,381.6,394.6,419.0,518.7,521.8,588.2,596.8,609.3,652.6,703.2,792.3$, $877.5,943.5,1096.7,1151.0,1197.9,1252.0,1276.6,1379.0,1486.3,1591.8,1711.2,1899.1$

dim-8 a: $23.6,40.4,44.4,64.8,106.1,113.8,364.3,382.4,426.8,438.6,494.1,496.0,587.9,590.1,602.0,602.6,614.2,615.1$, 863.6, 870.3, 1066.9, 1104.7, 1132.3, 1134.7, 1305.6, 1335.4, 1423.9, 1447.3, 3733.3, 3750.3

dim-8ts $\quad$ a: $1082.0 i, 40.4,78.7,86.7,118.5,210.2,283.0,284.1,334.6,344.5,468.2,491.9,502.7,573.7,585.6,627.2,703.0,739.6$, 899.3, 958.6, 1022.1, 1224.2, 1254.2, 1288.3, 1311.2, 1418.6, 1463.2, 1487.3, 1752.0, 1761.7

$\mathrm{CF}_{3} \mathrm{OH}-\mathrm{HF} \quad \mathrm{a}: 45.7,88.3,151.1,252.5,363.6,418.2,450.5,552.2,611.4,622.2,635.6,900.3,1124.8,1178.9,1327.0,1454.1,3724.4$, 4036.8

CF $_{3}$ OH-HFts $\quad$ a: $1434.8 i, 112.7,299.8,338.2,413.3,511.8,584.7,616.6,682.2,883.6,920.9,1075.8,1215.5,1299.2,1464.3,1617.1$, $1771.0,1840.2$

$\mathrm{CF}_{3} \mathrm{OH}-2 \mathrm{HF}$ a: $35.7,58.6,85.2,151.9,185.1,241.3,402.8,424.1,455.7,549.7,590.2,600.1,610.1,614.9,661.2,842.1,873.2,1061.9$, $1156.5,1352.1,1475.4,3543.9,3690.0,3836.0$

CF $_{3}$ OH-2HFts $\quad$ a: $1298.7 i, 51.1,95.0,135.7,285.6,319.8,383.6,465.3,565.7,600.6,634.3,657.7,809.3,934.4,1117.5,1163.2,1216.3$, $1260.9,1350.6,1463.4,1580.9,1655.1,1744.9,1929.1$

dim-HF a: $20.3,24.4,39.4,54.4,62.0,76.2,143.7,148.5,192.6,403.2,409.9,421.5,442.8,458.1,540.8,592.1,595.9,598.7$, 607.0, 614.1, 622.9, 684.8, 740.1, 859.2, 882.5, 1063.9, 1143.4, 1156.4, 1188.0, 1282.1, 1347.6, 1439.0, 1475.3, 3518.7, $3571.9,3850.4$ 


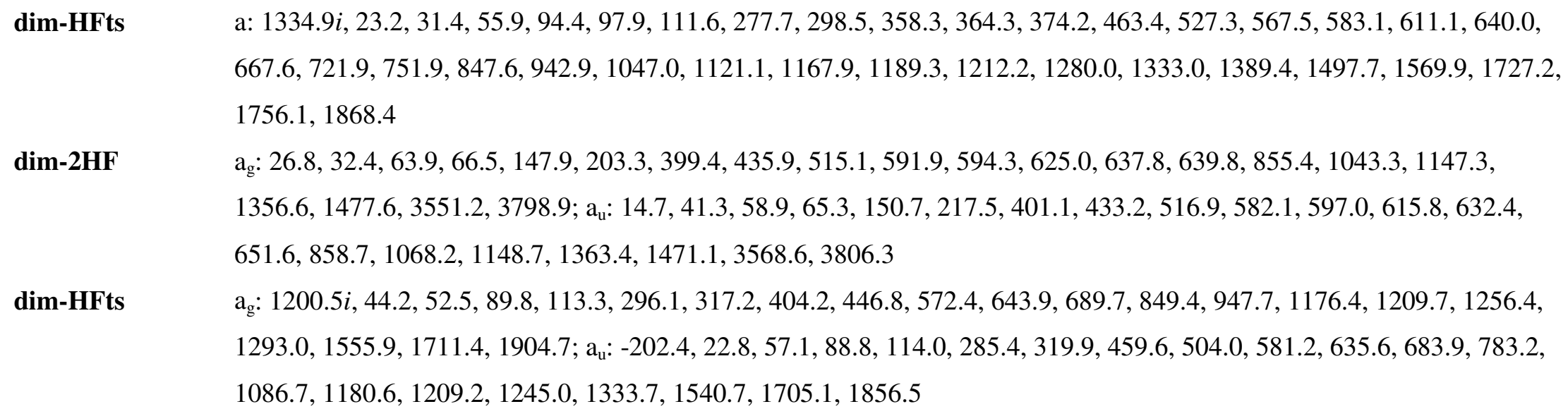

${ }^{\mathrm{a}} \mathrm{MP} 2 / \mathrm{aVTZ}$ results excepting for those of the $\mathrm{CF}_{3} \mathrm{OH}$ dimers and the $\mathrm{CF}_{3} \mathrm{OH}$ with $2 \mathrm{HF}$ systems obtained with $\mathrm{MP} 2 / \mathrm{aVDZ}$. 

Table S3. Tunneling corrections $\left(Q_{\text {tunnel }}\right)$ on the rate coefficients calculated using the Skodje \& Truhlar (ST) equations for the uncatalyzed reactions. ${ }^{a}$

\begin{tabular}{|c|c|c|c|c|c|}
\hline$\left.\right|_{T(\mathrm{~K})} ^{\text {Reaction }}\left(\omega_{\mathrm{i}}\right)$ & $\begin{array}{c}\mathrm{CF}_{3} \mathrm{OH} \rightarrow \\
\mathbf{C F}_{3} \mathbf{O H}-\mathbf{t s} \rightarrow \\
\mathrm{CF}_{2} \mathrm{O}+\mathrm{HF} \\
(1740.7 i)\end{array}$ & $\begin{array}{c}2 \mathrm{CF}_{3} \mathrm{OH} \rightarrow \\
\operatorname{dim}-6 \mathrm{ts} \rightarrow \\
\mathrm{CF}_{3} \mathrm{OH}+ \\
\mathrm{CF}_{2} \mathrm{O}+\mathrm{HF} \\
(1413.5 i)\end{array}$ & $\begin{array}{c}\operatorname{dim}-6 \rightarrow \\
\operatorname{dim}-6 \mathrm{ts} \rightarrow \\
\mathrm{CF}_{3} \mathrm{OH}+ \\
\mathrm{CF}_{2} \mathrm{O}+\mathrm{HF} \\
(1413.5 i)\end{array}$ & $\begin{array}{c}\operatorname{dim}-8 \rightarrow \\
\operatorname{dim}-8 t s \rightarrow 2 \\
\mathrm{CF}_{2} \mathrm{O}+2 \mathrm{HF} \\
\quad(1082.0 i)\end{array}$ & $\begin{array}{c}\operatorname{dim}-8 \rightarrow \\
\operatorname{dim}-8 t s \rightarrow 2 \\
\mathrm{CF}_{2} \mathrm{O}+(\mathrm{HF})_{2} \\
(1082.0 i)\end{array}$ \\
\hline 150 & $1.97 \times 10^{34}$ & $3.70 \times 10^{13}$ & $3.70 \times 10^{13}$ & $1.24 \times 10^{9}$ & $5.78 \times 10^{10}$ \\
\hline 200 & $5.41 \times 10^{20}$ & $3.33 \times 10^{7}$ & $3.33 \times 10^{7}$ & $8.00 \times 10^{3}$ & $3.28 \times 10^{4}$ \\
\hline 250 & $4.61 \times 10^{12}$ & $1.08 \times 10^{4}$ & $1.08 \times 10^{4}$ & $2.69 \times 10^{1}$ & $3.13 \times 10^{1}$ \\
\hline 260 & $2.71 \times 10^{11}$ & $3.33 \times 10^{3}$ & $3.33 \times 10^{3}$ & $1.57 \times 10^{1}$ & $1.68 \times 10^{1}$ \\
\hline 270 & $1.99 \times 10^{10}$ & $1.16 \times 10^{3}$ & $1.16 \times 10^{3}$ & $1.04 \times 10^{1}$ & $1.08 \times 10^{1}$ \\
\hline 280 & $1.77 \times 10^{9}$ & $4.56 \times 10^{2}$ & $4.56 \times 10^{2}$ & 7.67 & 7.78 \\
\hline 290 & $1.90 \times 10^{8}$ & $2.00 \times 10^{2}$ & $2.00 \times 10^{2}$ & 6.03 & 6.07 \\
\hline 298.15 & $3.46 \times 10^{7}$ & $1.10 \times 10^{2}$ & $1.10 \times 10^{2}$ & 5.15 & 5.16 \\
\hline 300 & $2.39 \times 10^{7}$ & $9.75 \times 10^{1}$ & $9.75 \times 10^{1}$ & 4.98 & 5.00 \\
\hline 310 & $3.49 \times 10^{6}$ & $5.28 \times 10^{1}$ & $5.28 \times 10^{1}$ & 4.26 & 4.27 \\
\hline 320 & $5.87 \times 10^{5}$ & $3.15 \times 10^{1}$ & $3.15 \times 10^{1}$ & 3.74 & 3.74 \\
\hline 330 & $1.12 \times 10^{5}$ & $2.05 \times 10^{1}$ & $2.05 \times 10^{1}$ & 3.35 & 3.35 \\
\hline 340 & $2.45 \times 10^{4}$ & $1.44 \times 10^{1}$ & $1.44 \times 10^{1}$ & 3.05 & 3.05 \\
\hline 350 & $6.03 \times 10^{3}$ & $1.08 \times 10^{1}$ & $1.08 \times 10^{1}$ & 2.80 & 2.81 \\
\hline 400 & $4.42 \times 10^{1}$ & 4.49 & 4.49 & 2.09 & 2.09 \\
\hline 450 & 7.93 & 2.93 & 2.93 & 1.75 & 1.75 \\
\hline 500 & 4.22 & 2.28 & 2.28 & 1.56 & 1.56 \\
\hline 600 & 2.40 & 1.71 & 1.71 & 1.35 & 1.35 \\
\hline 700 & 1.83 & 1.46 & 1.46 & 1.24 & 1.24 \\
\hline 800 & 1.57 & 1.33 & 1.33 & 1.18 & 1.18 \\
\hline 900 & 1.41 & 1.25 & 1.25 & 1.14 & 1.14 \\
\hline 1000 & 1.32 & 1.20 & 1.20 & 1.11 & 1.11 \\
\hline 1100 & 1.25 & 1.16 & 1.16 & 1.09 & 1.09 \\
\hline 1200 & 1.21 & 1.13 & 1.13 & 1.07 & 1.07 \\
\hline 1300 & 1.17 & 1.11 & 1.11 & 1.06 & 1.06 \\
\hline 1400 & 1.15 & 1.09 & 1.09 & 1.05 & 1.05 \\
\hline
\end{tabular}


${ }^{a}$ The imaginary frequency of $\mathrm{CF}_{3} \mathrm{OH}$-ts was taken from MP2/aVTZ calculations, and from MP2/aVDZ for the dimers. 
Table S4. Tunneling corrections $\left(Q_{\text {tunnel }}\right)$ on the rate coefficients calculated using the Skodje \& Truhlar (ST) equations for the $\mathrm{CF}_{3} \mathrm{OH}$ with $\mathrm{HF}$ and $2 \mathrm{HF}$ autocatalyzed reactions. ${ }^{\mathrm{a}}$

\begin{tabular}{|c|c|c|c|c|c|c|}
\hline $\int^{\text {Reaction }}\left(\omega_{\mathrm{i}}\right)$ & $\begin{array}{c}\mathrm{CF}_{3} \mathrm{OH}+ \\
\mathrm{HF} \rightarrow \\
\mathbf{C F}_{3} \mathrm{OH}- \\
\mathbf{H F t s} \rightarrow \\
\mathrm{CF}_{2} \mathrm{O}+2 \mathrm{HF} \\
(1434.8 i)\end{array}$ & $\begin{array}{c}\mathrm{CF}_{3} \mathrm{OH}+ \\
\mathrm{HF} \rightarrow \\
\mathbf{C F}_{3} \mathrm{OH}- \\
\mathbf{H F t s} \rightarrow \\
\mathrm{CF}_{2} \mathrm{O}+ \\
(\mathrm{HF})_{2} \\
(1434.8 i)\end{array}$ & $\begin{array}{c}\mathrm{CF}_{3} \mathrm{OH}-\mathrm{HF} \\
\rightarrow \mathrm{CF}_{3} \mathrm{OH}- \\
\mathrm{HFts} \rightarrow \\
\mathrm{CF}_{2} \mathrm{O}+2 \mathrm{HF} \\
(1434.8 i)\end{array}$ & $\begin{array}{c}\mathrm{CF}_{3} \mathrm{OH}-\mathrm{HF} \\
\rightarrow \mathrm{CF}_{3} \mathrm{OH}- \\
\mathbf{H F t s} \rightarrow \\
\mathrm{CF}_{2} \mathrm{O}+ \\
(\mathrm{HF})_{2} \\
(1434.8 i)\end{array}$ & $\begin{array}{c}\mathrm{CF}_{3} \mathrm{OH}+ \\
(\mathrm{HF})_{2} \rightarrow \\
\mathbf{C F}_{3} \mathrm{OH}- \\
\text { 2HFts } \rightarrow \\
\mathrm{CF}_{3} \mathrm{OH}+ \\
3 \mathrm{HF} \\
(1298.7 i)\end{array}$ & $\begin{array}{c}\mathrm{CF}_{3} \mathrm{OH}- \\
\mathbf{2 H F} \rightarrow \\
\mathbf{C F}_{3} \mathrm{OH}- \\
\mathbf{2 H F t s} \rightarrow \\
\mathrm{CF}_{3} \mathrm{OH}+ \\
3 \mathrm{HF} \\
(1298.7 i)\end{array}$ \\
\hline 150 & $2.92 \times 10^{12}$ & $5.79 \times 10^{14}$ & $5.17 \times 10^{15}$ & $1.02 \times 10^{18}$ & $9.76 \times 10^{3}$ & $4.86 \times 10^{8}$ \\
\hline 200 & $9.96 \times 10^{6}$ & $1.74 \times 10^{8}$ & $5.66 \times 10^{8}$ & $9.86 \times 10^{9}$ & $2.02 \times 10^{2}$ & $4.38 \times 10^{4}$ \\
\hline 250 & $7.01 \times 10^{3}$ & $2.84 \times 10^{4}$ & $5.07 \times 10^{4}$ & $2.05 \times 10^{5}$ & $2.60 \times 10^{1}$ & $2.55 \times 10^{2}$ \\
\hline 260 & $2.43 \times 10^{3}$ & $7.89 \times 10^{3}$ & $1.28 \times 10^{4}$ & $4.15 \times 10^{4}$ & $1.96 \times 10^{1}$ & $1.27 \times 10^{2}$ \\
\hline 270 & $9.41 \times 10^{2}$ & $2.48 \times 10^{3}$ & $3.70 \times 10^{3}$ & $9.74 \times 10^{3}$ & $1.53 \times 10^{1}$ & $6.93 \times 10^{1}$ \\
\hline 280 & $4.02 \times 10^{2}$ & $8.80 \times 10^{2}$ & $1.21 \times 10^{3}$ & $2.64 \times 10^{3}$ & $1.22 \times 10^{1}$ & $4.11 \times 10^{1}$ \\
\hline 290 & $1.90 \times 10^{2}$ & $3.50 \times 10^{2}$ & $4.50 \times 10^{2}$ & $8.22 \times 10^{2}$ & 9.93 & $2.63 \times 10^{1}$ \\
\hline 298.15 & $1.10 \times 10^{2}$ & $1.80 \times 10^{2}$ & $2.19 \times 10^{2}$ & $3.52 \times 10^{2}$ & 8.54 & $1.92 \times 10^{1}$ \\
\hline 300 & $9.81 \times 10^{1}$ & $1.56 \times 10^{2}$ & $1.89 \times 10^{2}$ & $2.95 \times 10^{2}$ & 8.28 & $1.80 \times 10^{1}$ \\
\hline 310 & $5.55 \times 10^{1}$ & $7.80 \times 10^{1}$ & $8.92 \times 10^{1}$ & $1.22 \times 10^{2}$ & 7.06 & $1.31 \times 10^{1}$ \\
\hline 320 & $3.40 \times 10^{1}$ & $4.33 \times 10^{1}$ & $4.75 \times 10^{1}$ & $5.85 \times 10^{1}$ & 6.13 & $1.00 \times 10^{1}$ \\
\hline 330 & $2.25 \times 10^{1}$ & $2.65 \times 10^{1}$ & $2.81 \times 10^{1}$ & $3.21 \times 10^{1}$ & 5.39 & 7.99 \\
\hline 340 & $1.59 \times 10^{1}$ & $1.77 \times 10^{1}$ & $1.84 \times 10^{1}$ & $1.99 \times 10^{1}$ & 4.81 & 6.59 \\
\hline 350 & $1.19 \times 10^{1}$ & $1.27 \times 10^{1}$ & $1.30 \times 10^{1}$ & $1.36 \times 10^{1}$ & 4.33 & 5.59 \\
\hline 400 & 4.80 & 4.83 & 4.84 & 4.86 & 2.93 & 3.23 \\
\hline 450 & 3.06 & 3.06 & 3.06 & 3.06 & 2.27 & 2.37 \\
\hline 500 & 2.35 & 2.35 & 2.35 & 2.35 & 1.91 & 1.96 \\
\hline 600 & 1.74 & 1.74 & 1.74 & 1.74 & 1.55 & 1.56 \\
\hline 700 & 1.48 & 1.48 & 1.48 & 1.48 & 1.37 & 1.37 \\
\hline 800 & 1.34 & 1.34 & 1.34 & 1.34 & 1.27 & 1.27 \\
\hline 900 & 1.26 & 1.26 & 1.26 & 1.26 & 1.20 & 1.21 \\
\hline 1000 & 1.20 & 1.20 & 1.20 & 1.20 & 1.16 & 1.16 \\
\hline 1100 & 1.16 & 1.16 & 1.16 & 1.16 & 1.13 & 1.13 \\
\hline
\end{tabular}




$\begin{array}{lllllll}1200 & 1.14 & 1.14 & 1.14 & 1.14 & 1.11 & 1.11 \\ 1300 & 1.11 & 1.11 & 1.11 & 1.11 & 1.09 & 1.09 \\ 1400 & 1.10 & 1.10 & 1.10 & 1.10 & 1.08 & 1.08 \\ 1500 & 1.08 & 1.08 & 1.08 & 1.08 & 1.06 & 1.07\end{array}$

a The imaginary frequency of $\mathrm{CF}_{3} \mathrm{OH}-\mathrm{HFts}$ was taken from MP2/aVTZ calculations, and that of $\mathrm{CF}_{3} \mathrm{OH}-2 \mathrm{HFts}$ from MP2/aVDZ for the dimers. 
Table S5. Tunneling corrections $\left(Q_{\text {tunnel }}\right)$ on the rate coefficients calculated using the Skodje \& Truhlar (ST) equations for the $\mathrm{CF}_{3} \mathrm{OH}$ dimer with $\mathrm{HF}$ autocatalyzed reactions. ${ }^{\text {a }}$

\begin{tabular}{|c|c|c|c|c|}
\hline $\begin{array}{r}\text { Reaction } \\
\left(\omega_{\mathrm{i}}\right)\end{array}$ & $\begin{array}{c}\text { dim-6 + HF } \rightarrow \\
\operatorname{dim}-\mathrm{HFts} \rightarrow \\
\mathrm{CF}_{3} \mathrm{OH}+\mathrm{CF}_{2} \mathrm{O}+ \\
2 \mathrm{HF} \\
(1334.9 i)\end{array}$ & $\begin{array}{c}\operatorname{dim}-6+\mathrm{HF} \rightarrow \\
\operatorname{dim}-\mathrm{HFts} \rightarrow \\
\mathrm{CF}_{3} \mathrm{OH}+\mathrm{CF}_{2} \mathrm{O}+ \\
(\mathrm{HF})_{2} \\
(1334.9 i)\end{array}$ & $\begin{array}{c}\text { dim- } \mathrm{HF} \rightarrow \text { dim- } \\
\text { HFts } \rightarrow \mathrm{CF}_{3} \mathrm{OH} \\
+\mathrm{CF}_{2} \mathrm{O}+2 \mathrm{HF} \\
(1334.9 i)\end{array}$ & $\begin{array}{c}\text { dim-HF } \rightarrow \text { dim- } \\
\text { HFts } \rightarrow \mathrm{CF}_{3} \mathrm{OH} \\
+\mathrm{CF}_{2} \mathrm{O}+(\mathrm{HF})_{2} \\
(1334.9 i)\end{array}$ \\
\hline 150 & $4.30 \times 10^{3}$ & $6.13 \times 10^{5}$ & $4.30 \times 10^{3}$ & $6.13 \times 10^{5}$ \\
\hline 200 & $1.43 \times 10^{2}$ & $1.82 \times 10^{3}$ & $1.43 \times 10^{2}$ & $1.82 \times 10^{3}$ \\
\hline 250 & $2.32 \times 10^{1}$ & $7.78 \times 10^{1}$ & $2.32 \times 10^{1}$ & $7.78 \times 10^{1}$ \\
\hline 260 & $1.80 \times 10^{1}$ & $5.05 \times 10^{1}$ & $1.80 \times 10^{1}$ & $5.05 \times 10^{1}$ \\
\hline 270 & $1.43 \times 10^{1}$ & $3.45 \times 10^{1}$ & $1.43 \times 10^{1}$ & $3.45 \times 10^{1}$ \\
\hline 280 & $1.16 \times 10^{1}$ & $2.46 \times 10^{1}$ & $1.16 \times 10^{1}$ & $2.46 \times 10^{1}$ \\
\hline 290 & 9.63 & $1.83 \times 10^{1}$ & 9.63 & $1.83 \times 10^{1}$ \\
\hline 298.15 & 8.37 & $1.47 \times 10^{1}$ & 8.37 & $1.47 \times 10^{1}$ \\
\hline 300 & 8.13 & $1.40 \times 10^{1}$ & 8.13 & $1.40 \times 10^{1}$ \\
\hline 310 & 6.98 & $1.11 \times 10^{1}$ & 6.98 & $1.11 \times 10^{1}$ \\
\hline 320 & 6.11 & 9.08 & 6.11 & 9.08 \\
\hline 330 & 5.41 & 7.59 & 5.41 & 7.59 \\
\hline 340 & 4.85 & 6.47 & 4.85 & 6.47 \\
\hline 350 & 4.39 & 5.62 & 4.39 & 5.62 \\
\hline 400 & 3.00 & 3.38 & 3.00 & 3.38 \\
\hline 450 & 2.33 & 2.49 & 2.33 & 2.49 \\
\hline 500 & 1.96 & 2.03 & 1.96 & 2.03 \\
\hline 600 & 1.57 & 1.60 & 1.57 & 1.60 \\
\hline 700 & 1.39 & 1.40 & 1.39 & 1.40 \\
\hline 800 & 1.28 & 1.29 & 1.28 & 1.29 \\
\hline 900 & 1.21 & 1.22 & 1.21 & 1.22 \\
\hline 1000 & 1.17 & 1.17 & 1.17 & 1.17 \\
\hline 1100 & 1.14 & 1.14 & 1.14 & 1.14 \\
\hline 1200 & 1.11 & 1.12 & 1.11 & 1.12 \\
\hline 1300 & 1.10 & 1.10 & 1.10 & 1.10 \\
\hline 1400 & 1.08 & 1.08 & 1.08 & 1.08 \\
\hline
\end{tabular}


${ }^{\mathrm{a}}$ The imaginary frequency was taken from MP2/aVDZ calculations. 
Table S6. Rate Constants at Different Temperatures with and without Tunneling Corrections.

\begin{tabular}{|c|c|c|c|c|}
\hline \multicolumn{5}{|c|}{$\mathrm{CF}_{3} \mathrm{OH} \rightarrow \mathrm{CF}_{2} \mathrm{O}+\mathrm{HF}$} \\
\hline$T$ & TST & $\mathrm{TST}^{*} Q$ & RRKM & $\operatorname{RRKM}^{*} Q$ \\
\hline 298.15 & $6.37 \times 10^{-21}$ & $2.20 \times 10^{-13}$ & $5.96 \times 10^{-21}$ & $2.06 \times 10^{-13}$ \\
\hline 500 & $2.03 \times 10^{-7}$ & $8.57 \times 10^{-7}$ & $1.88 \times 10^{-7}$ & $7.93 \times 10^{-7}$ \\
\hline 1000 & $2.13 \times 10^{3}$ & $2.81 \times 10^{3}$ & $1.49 \times 10^{3}$ & $1.96 \times 10^{3}$ \\
\hline \multicolumn{5}{|c|}{$\mathrm{CF}_{3} \mathrm{OH}+\mathrm{HF} \rightarrow \mathrm{CF}_{2} \mathrm{O}+2 \mathrm{HF}$} \\
\hline$T$ & TST & $\mathrm{TST}^{*} Q$ & & \\
\hline 298.15 & $1.09 \times 10^{-1}$ & $1.20 \times 10^{1}$ & & \\
\hline 500 & $4.72 \times 10^{3}$ & $1.11 \times 10^{4}$ & & \\
\hline 1000 & $2.09 \times 10^{7}$ & $2.51 \times 10^{7}$ & & \\
\hline \multicolumn{5}{|c|}{$\mathrm{CF}_{3} \mathrm{OH}+\mathrm{HF} \rightarrow \mathrm{CF}_{2} \mathrm{O}+(\mathrm{HF})_{2}$} \\
\hline$T$ & TST & $\mathrm{TST}^{*} Q$ & & \\
\hline 298.15 & $1.09 \times 10^{-1}$ & $1.96 \times 10^{-1}$ & & \\
\hline 500 & $4.72 \times 10^{3}$ & $1.11 \times 10^{4}$ & & \\
\hline 1000 & $2.09 \times 10^{7}$ & $2.51 \times 10^{7}$ & & \\
\hline \multicolumn{5}{|c|}{$\mathrm{CF}_{3} \mathrm{OH}-\mathrm{HF} \rightarrow \mathrm{CF}_{2} \mathrm{O}+2 \mathrm{HF}$} \\
\hline$T$ & TST & $\mathrm{TST}^{*} Q$ & RRKM & $\operatorname{RRKM}^{*} Q$ \\
\hline 298.15 & $3.45 \times 10^{-8}$ & $7.57 \times 10^{-6}$ & $3.98 \times 10^{-8}$ & $8.73 \times 10^{-6}$ \\
\hline 500 & 1.09 & 2.56 & 1.22 & 2.85 \\
\hline 1000 & $3.16 \times 10^{5}$ & $3.80 \times 10^{5}$ & $3.21 \times 10^{5}$ & $3.87 \times 10^{5}$ \\
\hline \multicolumn{5}{|c|}{$\mathrm{CF}_{3} \mathrm{OH}-\mathrm{HF} \rightarrow \mathrm{CF}_{2} \mathrm{O}+(\mathrm{HF})_{2}$} \\
\hline$T$ & TST & $\mathrm{TST}^{*} Q$ & RRKM & $\mathrm{RRKM}^{*} Q$ \\
\hline 298.15 & $3.45 \times 10^{-8}$ & $1.22 \times 10^{-5}$ & $3.98 \times 10^{-8}$ & $1.40 \times 10^{-5}$ \\
\hline 500 & 1.09 & 2.56 & 1.22 & 2.85 \\
\hline 1000 & $3.16 \times 10^{5}$ & $3.80 \times 10^{5}$ & $3.21 \times 10^{5}$ & $3.87 \times 10^{5}$ \\
\hline \multicolumn{5}{|c|}{$\mathrm{CF}_{3} \mathrm{OH}+(\mathrm{HF})_{2} \rightarrow \mathrm{CF}_{2} \mathrm{O}+3 \mathrm{HF}$} \\
\hline$T$ & TST & $\mathrm{TST}^{*} Q$ & & \\
\hline 298.15 & $2.09 \times 10^{5}$ & $1.79 \times 10^{6}$ & & \\
\hline 500 & $3.00 \times 10^{6}$ & $5.74 \times 10^{6}$ & & \\
\hline 1000 & $3.47 \times 10^{7}$ & $4.03 \times 10^{7}$ & & \\
\hline
\end{tabular}




\begin{tabular}{|c|c|c|c|c|}
\hline$T$ & TST & $\operatorname{TST}^{*} Q$ & RRKM & $\mathrm{RRKM}^{*} Q$ \\
\hline 298.15 & $6.84 \times 10^{-4}$ & $1.56 \times 10^{-2}$ & $7.74 \times 10^{-4}$ & $1.76 \times 10^{-2}$ \\
\hline 500 & $4.24 \times 10^{2}$ & $8.30 \times 10^{2}$ & $4.68 \times 10^{2}$ & $9.16 \times 10^{2}$ \\
\hline 1000 & $6.18 \times 10^{6}$ & $7.18 \times 10^{6}$ & $5.80 \times 10^{6}$ & $6.74 \times 10^{6}$ \\
\hline \multicolumn{5}{|c|}{$2 \mathrm{CF}_{3} \mathrm{OH} \rightarrow \mathrm{CF}_{2} \mathrm{O}+\mathrm{HF}+\mathrm{CF}_{3} \mathrm{OH}$} \\
\hline$T$ & $\mathrm{TST}$ & \multicolumn{2}{|c|}{$\mathrm{TST}^{*} Q$} & \\
\hline 298.15 & $1.17 \times 10^{-1}$ & \multicolumn{2}{|l|}{$1.29 \times 10^{1}$} & \\
\hline 500 & $2.93 \times 10^{3}$ & \multicolumn{2}{|l|}{$6.67 \times 10^{3}$} & \\
\hline 1000 & $1.29 \times 10^{7}$ & \multicolumn{2}{|l|}{$1.54 \times 10^{7}$} & \\
\hline \multicolumn{5}{|c|}{$\left(\mathrm{CF}_{3} \mathrm{OH}\right)_{2} \rightarrow \mathrm{CF}_{2} \mathrm{O}+\mathrm{HF}+\mathrm{CF}_{3} \mathrm{OH}$} \\
\hline$T$ & TST & $\mathrm{TST}^{*} Q$ & RRKM & $\mathrm{RRKM}^{*} Q$ \\
\hline 298.15 & $9.58 \times 10^{-11}$ & $1.06 \times 10^{-8}$ & $1.17 \times 10^{-10}$ & $1.29 \times 10^{-8}$ \\
\hline 500 & $2.42 \times 10^{-2}$ & $5.51 \times 10^{-2}$ & $2.79 \times 10^{-2}$ & $6.35 \times 10^{-2}$ \\
\hline 1000 & $3.79 \times 10^{4}$ & $4.53 \times 10^{4}$ & $4.10 \times 10^{4}$ & $4.90 \times 10^{4}$ \\
\hline \multicolumn{5}{|c|}{$2 \mathrm{CF}_{3} \mathrm{OH} \rightarrow 2 \mathrm{CF}_{2} \mathrm{O}+2 \mathrm{HF}$} \\
\hline$T$ & TST & $\mathrm{TST}^{*} Q$ & RRKM & $\mathrm{RRKM}^{*} Q$ \\
\hline 298.15 & $1.41 \times 10^{-13}$ & $7.26 \times 10^{-13}$ & $1.40 \times 10^{-13}$ & $7.20 \times 10^{-13}$ \\
\hline 500 & $9.68 \times 10^{-4}$ & $1.51 \times 10^{-3}$ & $9.57 \times 10^{-4}$ & $1.49 \times 10^{-3}$ \\
\hline 1000 & $1.84 \times 10^{4}$ & $2.04 \times 10^{4}$ & $1.80 \times 10^{4}$ & $2.00 \times 10^{4}$ \\
\hline \multicolumn{5}{|c|}{$2 \mathrm{CF}_{3} \mathrm{OH} \rightarrow 2 \mathrm{CF}_{2} \mathrm{O}+(\mathrm{HF})_{2}$} \\
\hline$T$ & TST & $\mathrm{TST}^{*} Q$ & RRKM & $\operatorname{RRKM}^{*} Q$ \\
\hline 298.15 & $1.41 \times 10^{-13}$ & $7.28 \times 10^{-13}$ & $1.40 \times 10^{-13}$ & $7.23 \times 10^{-13}$ \\
\hline 500 & $9.68 \times 10^{-4}$ & $1.51 \times 10^{-3}$ & $9.57 \times 10^{-4}$ & $1.49 \times 10^{-3}$ \\
\hline 1000 & $1.84 \times 10^{4}$ & $2.04 \times 10^{4}$ & $1.80 \times 10^{4}$ & $2.00 \times 10^{4}$ \\
\hline \multicolumn{5}{|c|}{$\left(\mathrm{CF}_{3} \mathrm{OH}\right)_{2} \rightarrow 2 \mathrm{CF}_{2} \mathrm{O}+2 \mathrm{HF}$} \\
\hline$T$ & TST & $\mathrm{TST}^{*} Q$ & RRKM & $\mathrm{RRKM}^{*} Q$ \\
\hline 298.15 & $1.41 \times 10^{-13}$ & $7.26 \times 10^{-13}$ & $1.40 \times 10^{-13}$ & $7.21 \times 10^{-13}$ \\
\hline 500 & $9.68 \times 10^{-4}$ & $1.51 \times 10^{-3}$ & $9.57 \times 10^{-4}$ & $1.49 \times 10^{-3}$ \\
\hline 1000 & $1.84 \times 10^{4}$ & $2.04 \times 10^{4}$ & $1.80 \times 10^{4}$ & $2.00 \times 10^{4}$ \\
\hline \multicolumn{5}{|c|}{$\left(\mathrm{CF}_{3} \mathrm{OH}\right)_{2} \rightarrow 2 \mathrm{CF}_{2} \mathrm{O}+(\mathrm{HF})_{2}$} \\
\hline$T$ & TST & $\mathrm{TST}^{*} Q$ & RRKM & $\mathrm{RRKM}^{*} Q$ \\
\hline 298.15 & $1.41 \times 10^{-13}$ & $7.28 \times 10^{-13}$ & $1.40 \times 10^{-13}$ & $7.23 \times 10^{-13}$ \\
\hline 500 & $9.68 \times 10^{-4}$ & $1.51 \times 10^{-3}$ & $9.57 \times 10^{-4}$ & $1.49 \times 10^{-3}$ \\
\hline
\end{tabular}




\begin{tabular}{|c|c|c|c|c|}
\hline 1000 & $1.84 \times 10^{4}$ & $2.04 \times 10^{4}$ & $1.80 \times 10^{4}$ & $2.00 \times 10^{4}$ \\
\hline \multicolumn{5}{|c|}{$\left(\mathrm{CF}_{3} \mathrm{OH}\right)_{2}+\mathrm{HF} \rightarrow \mathrm{CF}_{3} \mathrm{OH}+\mathrm{CF}_{2} \mathrm{O}+2 \mathrm{HF}$} \\
\hline$T$ & TST & $\mathrm{TST}^{*} Q$ & & \\
\hline 298.15 & $1.84 \times 10^{-2}$ & $1.54 \times 10^{-1}$ & & \\
\hline 500 & $3.70 \times 10^{2}$ & $7.24 \times 10^{2}$ & & \\
\hline 1000 & $8.81 \times 10^{5}$ & $1.03 \times 10^{6}$ & & \\
\hline \multicolumn{5}{|c|}{$\left(\mathrm{CF}_{3} \mathrm{OH}\right)_{2}+\mathrm{HF} \rightarrow \mathrm{CF}_{3} \mathrm{OH}+\mathrm{CF}_{2} \mathrm{O}+(\mathrm{HF})_{2}$} \\
\hline$T$ & $\mathrm{TST}$ & $\mathrm{TST}^{*} Q$ & & \\
\hline 298.15 & $1.84 \times 10^{-2}$ & $2.70 \times 10^{-1}$ & & \\
\hline 500 & $3.70 \times 10^{2}$ & $7.52 \times 10^{2}$ & & \\
\hline 1000 & $8.81 \times 10^{5}$ & $1.03 \times 10^{6}$ & & \\
\hline \multicolumn{5}{|c|}{$2 \mathrm{CF}_{3} \mathrm{OH}-\mathrm{HF} \rightarrow \mathrm{CF}_{3} \mathrm{OH}+\mathrm{CF}_{2} \mathrm{O}+2 \mathrm{HF}$} \\
\hline$T$ & TST & $\mathrm{TST}^{*} Q$ & RRKM & $\operatorname{RRKM}^{*} Q$ \\
\hline 298.15 & $4.24 \times 10^{-6}$ & $1.69 \times 10^{-4}$ & $4.87 \times 10^{-6}$ & $1.94 \times 10^{-4}$ \\
\hline 500 & $1.60 \times 10^{1}$ & $3.28 \times 10^{1}$ & $1.79 \times 10^{1}$ & $3.66 \times 10^{1}$ \\
\hline 1000 & $1.02 \times 10^{6}$ & $1.20 \times 10^{6}$ & $1.07 \times 10^{6}$ & $1.25 \times 10^{6}$ \\
\hline \multicolumn{5}{|c|}{$2 \mathrm{CF}_{3} \mathrm{OH}-\mathrm{HF} \rightarrow \mathrm{CF}_{3} \mathrm{OH}+\mathrm{CF}_{2} \mathrm{O}+(\mathrm{HF})_{2}$} \\
\hline$T$ & TST & $\mathrm{TST}^{*} Q$ & RRKM & $\mathrm{RRKM}^{*} Q$ \\
\hline 298.15 & $4.24 \times 10^{-6}$ & $2.13 \times 10^{-4}$ & $4.87 \times 10^{-6}$ & $2.45 \times 10^{-4}$ \\
\hline 500 & $1.60 \times 10^{1}$ & $3.28 \times 10^{1}$ & $1.79 \times 10^{1}$ & $3.66 \times 10^{1}$ \\
\hline 1000 & $1.02 \times 10^{6}$ & $1.20 \times 10^{6}$ & $1.07 \times 10^{6}$ & $1.25 \times 10^{6}$ \\
\hline
\end{tabular}


Table S7. Cartesian coordinates of MP2/aVTZ optimized geometries and symmetry.

$$
\begin{array}{lrrr}
\text { HF }\left({ }^{1} \Sigma_{\mathrm{g}}\right): C_{\infty \nu} \text { symmetry } \\
\text { F } & 0.000000 & 0.000000 & 0.092184 \\
\text { H } & 0.000000 & 0.000000 & -0.829654 \\
& & \\
\text { HF } & \text { dimer }\left({ }^{1} \mathrm{~A}^{\prime}\right): & C_{s} \text { symmetry } \\
\text { F } & 0.037992 & -1.310418 & 0.000000 \\
\text { H } & -0.824668 & -1.643968 & 0.000000 \\
\text { F } & 0.037992 & 1.435995 & 0.000000 \\
\text { H } & 0.140821 & 0.513770 & 0.000000
\end{array}
$$

$$
\begin{array}{lrcc}
\text { HF trimer }\left({ }^{1} \mathrm{~A}^{\prime}\right): & C_{3 h} \text { symmetry } \\
\text { F } & -0.939196 & 1.180545 & 0.000000 \\
\text { H } & 0.000000 & 1.179636 & 0.000000 \\
\text { F } & -0.552784 & -1.403640 & 0.000000 \\
\text { H } & -1.021595 & -0.589818 & 0.000000 \\
\text { F } & 1.491980 & 0.223095 & 0.000000 \\
\text { H } & 1.021595 & -0.589818 & 0.000000
\end{array}
$$

$\begin{array}{lccc}\mathrm{CF}_{3} \mathrm{OH}\left({ }^{1} \mathrm{~A}^{\prime}\right): & C_{s} \text { symmetry } \\ \mathrm{C} & 0.002618 & 0.021986 & 0.000000 \\ \mathrm{O} & -1.037732 & 0.876877 & 0.000000 \\ \mathrm{H} & -1.854371 & 0.362386 & 0.000000 \\ \mathrm{~F} & 1.121489 & 0.727759 & 0.000000 \\ \mathrm{~F} & 0.002618 & -0.781064 & 1.076080 \\ \mathrm{~F} & 0.002618 & -0.781064 & -1.076080\end{array}$

$$
\begin{array}{llll}
\mathrm{CF}_{3} \mathrm{O}^{\circ}\left({ }^{2} \mathrm{~A}^{\prime}\right): C_{s} \text { symmetry } \\
\mathrm{C} & -0.021721 & 0.025795 & 0.000000 \\
\mathrm{O} & -0.446853 & 1.319543 & 0.000000 \\
\mathrm{~F} & 1.305390 & 0.076370 & 0.000000 \\
\mathrm{~F} & -0.446853 & -0.633247 & 1.072845 \\
\mathrm{~F} & -0.446853 & -0.633247 & -1.072845
\end{array}
$$




$$
\begin{array}{llll}
\mathrm{CF}_{3} \mathrm{O}^{+}\left({ }^{3} \mathrm{~A}_{1}\right): C_{3 v} \text { symmetry } \\
\mathrm{C} & 0.000000 & 0.000000 & -0.381621 \\
\mathrm{O} & 0.000000 & 0.000000 & 1.857786 \\
\mathrm{~F} & 0.000000 & 1.237273 & -0.46570 \\
\mathrm{~F} & 1.071510 & -0.618636 & -0.46560 \\
\mathrm{~F} & -1.071510 & -0.618636 & -0.46560
\end{array}
$$

$\mathrm{CF}_{3} \mathrm{O}^{-}\left({ }^{1} \mathrm{~A}_{1}\right): C_{3 v}$ symmetry

$\begin{array}{llll}\text { C } & 0.000000 & 0.000000 & 0.190971\end{array}$

$\begin{array}{llll}\mathrm{O} & 0.000000 & 0.000000 & 1.410945\end{array}$

F $\quad 0.000000 \quad 1.268870 \quad-0.460496$

F $1.098873 \quad-0.634435 \quad-0.460496$

F -1.098873 $-0.634435 \quad-0.460496$

$\mathrm{CF}_{3} \mathrm{OH}^{\cdot+}\left({ }^{2} \mathrm{~A} "\right): C_{s}$ symmetry

$\begin{array}{llll}\text { C } & 0.186829 & -0.032233 & 0.000000\end{array}$

$\begin{array}{llll}\text { O } & -1.388805 & 0.456582 & 0.000000\end{array}$

H $-2.002150 \quad-0.326773 \quad 0.000000$

F $\quad 0.808555 \quad 1.072258 \quad 0.000000$

F $\quad 0.261908 \quad-0.709754 \quad 1.081733$

$\begin{array}{llll}\text { F } & 0.261908 & -0.709754 & -1.081733\end{array}$

$\mathrm{CF}_{3} \mathrm{OH}_{2}{ }^{+}\left({ }^{1} \mathrm{~A}^{\prime}\right): C_{s}$ symmetry

$\begin{array}{llll}\text { C } & 0.174670 & -0.008893 & 0.000000\end{array}$

O $-1.371601 \quad-0.072742 \quad 0.000000$

$\begin{array}{llll}\text { F } & 0.498817 & 1.241746 & 0.000000\end{array}$

F $\quad 0.498817 \quad-0.621163 \quad 1.082243$

F $0.498817-0.621163 \quad-1.082243$

H $-1.771628 \quad 0.320262-0.805293$

H $-1.771628 \quad 0.320262 \quad 0.805293$

$\mathrm{HF}-\mathrm{CF}_{2} \mathrm{OH}^{+}\left({ }^{1} \mathrm{~A}\right): C_{1}$ symmetry

$\begin{array}{llll}\text { C } & -0.564732 & -0.002233 & 0.003202\end{array}$ 
$\begin{array}{cccc}\text { O } & -0.607023 & 1.147012 & -0.485159 \\ \text { F } & 1.834769 & 0.034750 & -0.058159 \\ \text { F } & -0.499631 & -0.234721 & 1.235318 \\ \text { F } & -0.659809 & -0.985002 & -0.757732 \\ \text { H } & -0.507526 & 1.864648 & 0.176904 \\ \text { H } & 2.674155 & -0.362590 & -0.089682\end{array}$

$\mathrm{CF}_{2} \mathrm{OH}^{+}-\mathrm{FH}\left({ }^{1} \mathrm{~A}^{\prime}\right): C_{\mathrm{s}}$ symmetry

$\begin{array}{llll}\text { C } & 0.000000 & 0.764673 & 0.000000\end{array}$

$\begin{array}{llll}\text { O } & -0.883411 & -0.101098 & 0.000000\end{array}$

$\begin{array}{llll}\text { F } & -0.309445 & 1.979208 & 0.000000\end{array}$

F $\quad 1.236602 \quad 0.510434 \quad 0.000000$

H $-0.553012 \quad-1.0721390 .000000$

F $\quad-0.023564 \quad-2.431701 \quad 0.000000$

H $-0.512041 \quad-3.228586 \quad 0.000000$

$\mathrm{CF}_{3}\left({ }^{2} \mathrm{~A}_{1}\right): C_{3 v}$ symmetry

$\begin{array}{llll}\text { C } & 0.000000 & 0.000000 & 0.326451\end{array}$

F $\quad 0.000000 \quad 1.255404 \quad-0.072545$

F - $1.087212-0.627702 \quad-0.072545$

F $1.087212-0.627702 \quad-0.072545$

$\mathrm{CF}_{2} \mathrm{O}\left({ }^{1} \mathrm{~A}_{1}\right): C_{2 v}$ symmetry

$\begin{array}{llll}\text { C } & 0.000000 & 0.000000 & 0.143439\end{array}$

$\begin{array}{llll}\text { O } & 0.000000 & 0.000000 & 1.321250\end{array}$

$\begin{array}{llll}\text { F } & 0.000000 & 1.061711 & -0.635035\end{array}$

$\begin{array}{llll}\text { F } & 0.000000 & -1.061711 & -0.635035\end{array}$

$\mathrm{CF}_{2} \mathrm{O}^{\cdot+}\left({ }^{2} \mathrm{~B}_{2}\right): C_{2 v}$ symmetry

$\begin{array}{llll}\text { C } & 0.000000 & 0.000000 & 0.027310\end{array}$

$\begin{array}{lllll}\text { O } & 0.000000 & 0.000000 & 1.299369\end{array}$

F $\quad 0.000000 \quad 1.079135 \quad-0.586601$

F $\quad 0.000000 \quad-1.079135 \quad-0.586601$ 


$$
\begin{array}{lrrr}
\mathrm{CF}_{3} \mathrm{OH}-\mathrm{ts}\left({ }^{1} \mathrm{~A}^{\prime}\right): C_{s} \text { symmetry } \\
\mathrm{C} & 0.116224 & 0.251355 & 0.000000 \\
\mathrm{O} & 1.308606 & -0.148816 & 0.000000 \\
\mathrm{H} & 0.750722 & -1.225673 & 0.000000 \\
\mathrm{~F} & -0.441367 & -1.400919 & 0.000000 \\
\mathrm{~F} & -0.441367 & 0.750908 & 1.063417 \\
\mathrm{~F} & -0.441367 & 0.750908 & -1.063417
\end{array}
$$

$\mathrm{CHF}_{2} \mathrm{OH}\left({ }^{1} \mathrm{~A}^{\prime}\right): C_{s}$ symmetry

$$
\begin{array}{cccc}
\text { C } & 0.282882 & 0.218802 & 0.000000 \\
\text { O } & -0.798104 & 1.032524 & 0.000000 \\
\text { H } & -1.591168 & 0.479643 & 0.000000 \\
\text { H } & 1.186842 & 0.817705 & 0.000000 \\
\text { F } & 0.282882 & -0.603909 & 1.083353 \\
\text { F } & 0.282882 & -0.603909 & -1.083353
\end{array}
$$

$\mathrm{CHF}_{2} \mathrm{OH}$-ts $\left({ }^{1} \mathrm{~A}\right): C_{1}$ symmetry
$\begin{array}{llll}\text { C } & 0.280908 & 0.150049 & 0.395065\end{array}$
$\begin{array}{llll}\text { O } & -0.181187 & 1.198602 & -0.142963\end{array}$
H $-1.105446 \quad 0.460688 \quad-0.361770$
F $-1.188423 \quad-0.713165 \quad-0.057790$
F $1.256368 \quad-0.496929-0.200566$
H $0.257994 \quad-0.058959 \quad 1.460293$

$\mathrm{CH}_{2} \mathrm{FOH}\left({ }^{1} \mathrm{~A}\right): C_{1}$ symmetry

$\begin{array}{llll}\text { C } & 0.000000 & 0.000000 & 0.000000\end{array}$

$\begin{array}{lllll}\mathrm{O} & 0.000000 & 0.000000 & 1.379534\end{array}$

H $\quad 0.912417 \quad 0.000000 \quad 1.687906$

F $\quad 0.413157 \quad-1.229950 \quad-0.493871$

H $-1.0245610 .157019-0.321669$

H $\quad 0.678607 \quad 0.741144 \quad-0.421591$

$\mathrm{CH}_{2} \mathrm{FOH}-\mathrm{ts}\left({ }^{1} \mathrm{~A}\right): C_{1}$ symmetry

$\begin{array}{llll}\text { C } & 0.402958 & 0.638629 & 0.000000\end{array}$ 

$\begin{array}{llll}\text { O } & 0.971061 & -0.515401 & 0.000000\end{array}$
H $-0.221969 \quad-0.844027 \quad 0.000000$
F -1.177670 $-0.142107 \quad 0.000000$
H $\quad 0.317382 \quad 1.207212 \quad 0.926874$
H $\quad 0.317382 \quad 1.207212 \quad-0.926874$

dim-6: $C_{1}$ symmetry
$\begin{array}{llll}\text { C } & 2.154318 & 0.038022 & 0.009087\end{array}$
$\begin{array}{llll}\text { O } & 1.667213 & 1.269248 & -0.166864\end{array}$
H $\quad 0.698077 \quad 1.204724 \quad-0.187311$
F $1.704592 \quad-0.830654 \quad-0.936317$
$\begin{array}{llll}\text { F } & 3.473095 & 0.073925 & -0.065973\end{array}$
F $1.807652 \quad-0.500533 \quad 1.189264$
H $-0.599441 \quad-0.672224 \quad-0.907909$
$\begin{array}{llll}\text { O } & -0.927917 & 0.181346 & -0.589952\end{array}$
$\begin{array}{llll}\text { C } & -2.138040 & 0.009938 & -0.002521\end{array}$
F -2.539116 $1.185993 \quad 0.449811$
F $-3.045160-0.457889-0.864531$
F $-2.080025 \quad-0.851400 \quad 1.017785$

dim-6ts: $C_{1}$ symmetry
C $-1.905396 \quad-0.234551 \quad-0.021446$
$\begin{array}{lllll}\text { O } & -1.229539 & -1.026417 & -0.701287\end{array}$
H $-0.072469-0.626793 \quad-0.793530$
F -1.102469 $1.299919-0.147666$
F $-3.084070 \quad 0.131770 \quad-0.439755$
F -1.900719 $-0.305942 \quad 1.289574$
H $-0.022942 \quad 0.939791-0.477917$
$\begin{array}{llll}\text { O } & 0.782826 & 0.185582 & -0.856819\end{array}$
$\begin{array}{llll}\text { C } & 1.827669 & -0.014052 & -0.006986\end{array}$
F $2.589700 \quad 1.070633 \quad 0.031833$
F $1.404603 \quad-0.274553 \quad 1.234953$
$\begin{array}{llll}\text { F } & 2.552453 & -1.043460 & -0.423729\end{array}$ 
dim-8: $C_{1}$ symmetry (close to $C_{i}$ symmetry)

$$
\begin{array}{lrrr}
\text { C } & -1.935353 & 0.041811 & -0.004673 \\
\text { O } & -1.562010 & 1.316908 & 0.118381 \\
\text { H } & -0.652392 & 1.403254 & -0.204082 \\
\text { F } & -1.162459 & -0.793881 & 0.753834 \\
\text { F } & -3.181216 & -0.091140 & 0.405756 \\
\text { F } & -1.836088 & -0.420051 & -1.258417 \\
\text { H } & 0.651111 & -1.402299 & 0.202420 \\
\text { O } & 1.560829 & -1.316444 & -0.119910 \\
\text { C } & 1.935327 & -0.041825 & 0.004634 \\
\text { F } & 1.162687 & 0.795506 & -0.752293 \\
\text { F } & 1.837218 & 0.418444 & 1.259041 \\
\text { F } & 3.181068 & 0.090612 & -0.406351
\end{array}
$$

dim-8ts: $C_{1}$ symmetry (close to $C_{i}$ symmetry)
$\begin{array}{llll}\text { C } & 1.814553 & 0.231408 & -0.017020\end{array}$
$\begin{array}{llll}\text { O } & 1.109843 & 1.050251 & -0.620338\end{array}$
H $\quad 0.039505 \quad 1.243182 \quad-0.158976$
F $1.029451-1.333842 \quad-0.210356$
F $2.960127-0.109324 \quad-0.522894$
F $1.855563 \quad 0.209745 \quad 1.293813$
H $-0.038702 \quad-1.240729 \quad 0.179743$
$\begin{array}{llll}\text { O } & -1.113450 & -1.034858 & 0.647925\end{array}$
$\begin{array}{llll}\text { C }-1.808140 & -0.224951 & 0.022441\end{array}$
$\begin{array}{llll}\text { F } & -1.051236 & 1.330101 & 0.205463\end{array}$
F -1.823797 $-0.224794-1.293749$
F $-2.971265 \quad 0.109855 \quad 0.497280$

$\mathrm{CF}_{3} \mathrm{OH}-\mathrm{HF}: C_{1}$ symmetry
$\begin{array}{llll}\text { C } & 0.608299 & 0.042281 & 0.000806\end{array}$
O $\quad 0.020926 \quad 1.232507 \quad-0.145996$
$\begin{array}{llll}\mathrm{H} & -0.925246 & 1.126854 & 0.045478\end{array}$
F $\quad 1.869828 \quad 0.126459-0.383236$
F $\quad 0.575463 \quad-0.409970 \quad 1.263449$ 


$$
\begin{array}{llll}
\text { H } & -2.177194 & -0.731675 & -0.345190 \\
\text { F } & -2.531526 & 0.037715 & 0.032317 \\
\text { F } & 0.006817 & -0.921861 & -0.749992
\end{array}
$$

\section{$\mathrm{CF}_{3} \mathrm{OH}$-HFts: $C_{1}$ symmetry}

$$
\begin{array}{lrrr}
\text { C } & -0.612186 & -0.222173 & -0.003057 \\
\text { O } & 0.144195 & -1.156270 & -0.326209 \\
\text { H } & 1.261800 & -0.837593 & -0.102630 \\
\text { F } & -1.600544 & 0.102564 & -0.780220 \\
\text { F } & -0.939235 & -0.024496 & 1.249751 \\
\text { H } & 1.389769 & 0.732774 & -0.078829 \\
\text { F } & 2.163135 & -0.110291 & 0.027271 \\
\text { F } & 0.361976 & 1.219780 & -0.184638
\end{array}
$$

$\mathrm{CF}_{3} \mathrm{OH}-2 \mathrm{HF}$ : $C_{1}$ symmetry

$$
\begin{array}{lrrr}
\text { C } & 1.018902 & 0.038339 & -0.005108 \\
\text { O } & 0.652701 & 1.186534 & -0.551636 \\
\text { H } & -0.286567 & 1.361376 & -0.340521 \\
\text { F } & 2.309572 & -0.160340 & -0.183473 \\
\text { F } & 0.735728 & -0.053612 & 1.301608 \\
\text { F } & 0.372857 & -1.042712 & -0.581669 \\
\text { F } & -2.241889 & -1.221459 & -0.007167 \\
\text { H } & -1.344301 & -1.402708 & -0.196621 \\
\text { F } & -2.000958 & 1.351973 & 0.018866 \\
\text { H } & -2.281946 & 0.454372 & 0.047403
\end{array}
$$

\section{$\mathrm{CF}_{3}$ OH-2HFts: $C_{1}$ symmetry}

$$
\begin{array}{lrrr}
\text { C } & -0.990382 & -0.234364 & -0.001721 \\
\text { O } & -0.359132 & -1.060037 & -0.676871 \\
\text { H } & 0.729354 & -1.222362 & -0.319171 \\
\text { F } & -2.183231 & 0.100555 & -0.373538 \\
\text { F } & -0.867533 & -0.194544 & 1.300315 \\
\text { F } & -0.237960 & 1.337972 & -0.303822 \\
\text { F } & 1.995267 & 1.060324 & 0.030662
\end{array}
$$




$$
\begin{array}{llll}
\mathrm{H} & 0.873089 & 1.271219 & -0.142678 \\
\mathrm{~F} & 1.867722 & -1.202051 & -0.002961 \\
\mathrm{H} & 2.044518 & -0.082683 & 0.031229
\end{array}
$$

dim-HF: $C_{1}$ symmetry

$$
\begin{array}{llll}
\text { C } & 2.288743 & -0.271991 & -0.001765 \\
\text { O } & 1.031790 & 0.105547 & -0.321941 \\
\text { H } & 0.903183 & 1.062167 & -0.169074 \\
\text { F } & 2.576837 & -0.017275 & 1.278487 \\
\text { F } & 2.392832 & -1.576116 & -0.210800 \\
\text { F } & 3.201935 & 0.358327 & -0.745996 \\
\text { H } & -0.515256 & -0.810309 & -0.615443 \\
\text { O } & -1.437004 & -1.092672 & -0.772055 \\
\text { C } & -2.250308 & -0.375713 & -0.009797 \\
\text { F } & -3.484165 & -0.826667 & -0.114390 \\
\text { F } & -1.897327 & -0.353971 & 1.281708 \\
\text { F } & -2.282817 & 0.951233 & -0.393345 \\
\text { F } & -0.106758 & 2.502504 & -0.017243 \\
\text { H } & -0.981653 & 2.189050 & -0.099933
\end{array}
$$

dim-HFts: $C_{1}$ symmetry

$$
\begin{array}{lrrr}
\text { C } & -2.122398 & -0.224766 & -0.000488 \\
\text { O } & -0.919117 & 0.062532 & -0.565851 \\
\text { H } & -0.531857 & 1.124817 & -0.352427 \\
\text { F } & -2.093119 & -0.068474 & 1.325437 \\
\text { F } & -3.055786 & 0.578282 & -0.493256 \\
\text { F } & -2.444200 & -1.485414 & -0.263114 \\
\text { H } & -0.008058 & -0.587863 & -0.153447 \\
\text { O } & 0.991751 & -1.064621 & 0.329533 \\
\text { C } & 2.042497 & -0.534121 & -0.047462 \\
\text { F } & 3.167644 & -0.895553 & 0.483536 \\
\text { F } & 2.204783 & -0.182104 & -1.301505 \\
\text { F } & 2.039498 & 1.111846 & 0.601597 \\
\text { F } & 0.107443 & 2.093557 & -0.085479
\end{array}
$$


$\begin{array}{llll}\mathrm{H} & 1.101875 & 1.663811 & 0.279173\end{array}$

$\operatorname{dim}-2 \mathrm{HF}\left({ }^{1} \mathrm{~A}_{\mathrm{g}}\right): C_{i}$ symmetry

$\begin{array}{llll}\text { C } & -2.238941 & 0.666846 & -0.650047 \\ \text { O } & -2.721261 & 0.871097 & 0.561520 \\ \text { H } & -1.980724 & 0.921008 & 1.197935 \\ \text { F } & -1.701662 & -0.601884 & -0.787272 \\ \text { F } & -3.196420 & 0.769947 & -1.550652 \\ \text { F } & -1.234195 & 1.494651 & -0.982330 \\ \text { H } & 1.980724 & -0.921008 & -1.197935 \\ \text { O } & 2.721261 & -0.871097 & -0.561520 \\ \text { C } & 2.238941 & -0.666846 & 0.650047 \\ \text { F } & 1.701662 & 0.601884 & 0.787272 \\ \text { F } & 1.234195 & -1.494651 & 0.982330 \\ \text { F } & 3.196420 & -0.769947 & 1.550652 \\ \text { H } & -0.275442 & -0.865078 & -1.761518 \\ \text { F } & 0.548900 & -1.002168 & -2.181304 \\ \text { H } & 0.275442 & 0.865078 & 1.761518 \\ \text { F } & -0.548900 & 1.002168 & 2.181304\end{array}$

dim-2HFts $\left({ }^{1} \mathrm{~A}_{\mathrm{g}}\right): C_{i}$ symmetry
$\begin{array}{llll}\text { C } & -1.805000 & 1.436103 & -0.300723\end{array}$
$\begin{array}{llll}\mathrm{O} & -1.812360 & 1.531010 & 0.928828\end{array}$
$\mathrm{H} \quad-0.779063 \quad 1.363381 \quad 1.437696$
F $\quad-1.832727 \quad-0.299786 \quad-0.630479$
F $\quad-2.874678 \quad 1.737796 \quad-0.967725$
F $\quad-0.723222 \quad 1.700097 \quad-0.995420$
$\mathrm{H} \quad 0.779063 \quad-1.363381-1.437696$
O $\quad \begin{array}{llll}1.812360 & -1.531010 & -0.928828\end{array}$
$\begin{array}{llll}\text { C } & 1.805000 & -1.436103 & 0.300723\end{array}$
F $\quad 1.832727 \quad 0.299786 \quad 0.630479$
F $\quad 0.723222 \quad-1.700097 \quad 0.995420$
F $\quad 2.874678 \quad-1.737796 \quad 0.967725$
$\begin{array}{llll}\mathrm{H} & -1.045784 & -0.732612 & -1.314885\end{array}$ 

F $\quad-0.239464 \quad-1.190791 \quad-1.977087$
$\begin{array}{llll}\mathrm{H} & 1.045784 & 0.732612 & 1.314885\end{array}$
F $\quad 0.239464 \quad 1.190791 \quad 1.977087$ 